\title{
Social Stratification, Gender and Sport Participation
}

\author{
by Aaron Reeves \\ University of Essex
}

\author{
Sociological Research Online, 17 (2) 12 \\ <http://www.socresonline.org.uk/17/2/12.html> \\ $10.5153 /$ sro. 2598
}

Received: 2 Nov 2011 Accepted: 6 Feb 2012 Published: 31 May 2012

\begin{abstract}
Correlations between social class and sport participation have frequently been observed (Crook 1997; Ceron-Anaya 2010; Dollman and Lewis 2010; Stalsberg and Pedersen 2010). However, discrete associations between occupational class positions and specific sporting activities overlook the complex interrelationships amongst these sports. Until recently understanding the relationality of sport has been constrained by a lack of available and appropriate data. Work by Bourdieu (1984), and more recently Bennett et al. (2009), have explored the general field of cultural consumption and sport has been one dimension of these treatments. Using multiple correspondence analysis (Le Roux and Rouanet 2004), this research focuses upon the social space of sport participation in Britain in order to provide a more detailed account of how these activities are organised. From data in the Taking-Part Survey $(n=10,349)$, which was gathered between July 2005-October 2006, 19 sporting practices are situated along four key dimensions. The first dimension separates gender and corresponds to a division between an embodied or social focus. Dimension two captures the impact of age. Internal and external orientations divide dimension three, where men tend to be internally oriented. Class, education and social status are significant along this dimension. Dimension four differentiates self-employed and manual workers; reinforcing occupational and educational differences. Consequently, the social space of sports participation cannot be neatly contained within the logic of class; other explanations drawing on friendship, education and embodiment are also required.
\end{abstract}

\section{Keywords: Bourdieu, Sport, Culture, Class, Gender, Education}

\section{Introduction}

1.1 Over thirty years ago, Pierre Bourdieu (1978) argued that sport is an important locus of conflict between social classes. In 2004 Rowe et al. concluded, following failures to increase sporting participation amongst Britons (despite government funding), that sport is a primary factor in the reproduction of class differences (Rowe et al. 2004). Similar trends have been observed in other countries (Crook 1997; Dollman and Lewis 2010; Stalsberg and Pedersen 2010) and in specific sports, for example Golf (Ceron-Anaya 2010). These issues require careful attention because of the economic and cultural centrality of sport in Western societies (Washington and Karen 2001) and its influence on politics and nationhood globally (Cha 2009); and yet, what these correlations mean is not always apparent. Sport, for Bennett et al., is 'an issue of status and social classification in its own right' (Bennett 2009: 155) while for Warde (2006), in conjunction with Bennett et al.'s research, class has very little effect upon sporting participation. The British government have continued to use public funds to analyse sporting participation whilst promoting physical activity among the populace (DCMS 2010); understanding these patterns is important for a government preparing for the 2012 Olympics and concerned with increasing health service costs (Mays 2008; DoH 2009).

1.2 Following Bourdieu (1984), Bennett et al.'s study of 'Culture, Class, Distinction' (2009) surveys the field of cultural consumption using multiple correspondence analysis (MCA). Reproducing Bourdieu's research in a different context re-examines the relevance of his thesis for understanding contemporary cultural consumption, leisure and social stratification (see also Lahire 2010). Although Bennett et al.'s general survey of cultural consumption and leisure is invaluable it did not intend to present a detailed analysis of every possible field of activity. Building upon this broad approach to leisure consumption, this research will specifically provide a detailed analysis of the primary dimensions which structure the social space of sports participation whilst also illuminating important connections between sport and the broader field of leisure. ${ }^{[1]}$ Further, because the dimensions in this research demonstrate the significance of gender, age and social stratification, dimensions which are also notable in Bennett et al.'s work, this illumination is especially prescient. 
1.3 For Bourdieu each field of practice (such as literature, music or sport) is structured by an autonomous logic which is embedded within more general dispositions forming homologies between fields of practice and the social strata. Each field, therefore, should be studied in its own right (Bourdieu 2008 [1999]) and from these studies inferences can be made regarding the structure of social relations. This 'homology thesis' has been reinvigorated in recent research (Lopez-Sintas and Garcia-Alvarez 2006; Bennett et al. 2009) whilst also garnering some additional criticism (Coulangeon 2004; Coulangeon and Lemel 2009).

1.4 This research will unpack those dynamics which structure the field of sport participation. One such dynamic is the connection between class, morality and the Self which provide a useful framework for considering the role of sport in these classificatory practices (Lamont 1992). Sport serves a variety of functions, namely: training of mind-body, generation of ethical values and attaining well-being (Bennett et al. 2009; Ceron-Anaya 2010). These same notions are closely tied with the rhetoric of class (Skeggs 2004a) and therefore serve as markers of stratification; 'the chief bourgeois characteristics against which the working class, and indeed the gentry, are judged... is self-mastery' (Day 2001: 137). Practices that signify self-mastery are therefore anticipated to be correlated with class divisions in this research.

1.5 Interest in sport can be grounded in training the body. If 'the body is the most indisputable materialization of class taste' (Bourdieu 1984: 190) then the assocaition with sporting practices should be anticipated. Yet, as Bourdieu elaborates, trying to unpack these intersections is problematic. Although he draws attention to an instrumental relationship toward the body, primarily expressed by the working classes, there is no description, detailed or otherwise, referring to how women and men may differ within these class positions regarding a) their relation to the body and b) how this relation is manifest in practice. Rather, there are only broadly conceived distinctions between the instrumental working class habitus and the middle class tendency to 'cultivate the body... with an ascetic exaltation of controlled diet' (Bourdieu 1984: 213). In addition the dominant class, Bourdieu argues, 'treats [the] body as an end... a sign of its own ease' (1984: 218). This dominant group select practices that afford exclusivity in place and persons, and low physicality.

1.6 Self-mastery is hypothesised to be a key component of the social space of sport participation and yet self-mastery will also be mediated by gender. Bourdieu's failure to examine the role of gender in each of these dimensions is a noteworthy neglect. Criticism of this lack has been outlined elsewhere and so will not be discussed in detail here (McNay 1999; Adkins and Skeggs 2004; Skeggs 2004b; Krais 2006). Yet, it will be fruitful to consider Brown's (2006) neo-Bourdieusian approach to this issue within the context of this research. Brown posits that, for Bourdieu (2001), there is an assumed gendered multiplicity which recognises the variety of positions an individual can take in relation to gender (for example, homosexual men may try to distance themselves from certain forms of masculinity). This multiplicity is also constrained (to an extent) by the sex of the subject; therefore gay men will still position themselves in relation to other males and may benefit from being male in certain settings (Connell 1995; Connell and Messerschmidt 2005; Brown 2006). In this view, dispositions of class and gender increasingly intersect and conflict (Anthias 2005; Law 2007).

1.7 In this regard, a number of patterns in Bennett et al. (2009) are reminiscent of Bourdieu's earlier account. Their MCA demonstrates that sport is a major contributor to the dimension correlated with gender, though this is primarily an axis concerned with taste (Bennett et al. 2009: 47; see also fig. 3.7). Accordingly it is important to examine whether sporting practices, as opposed to preference for a sport, are divided along similar criteria. From a slightly different perspective Bunting et al. (2008) have observed that women are more likely to attend cultural events than men. This division in cultural orientation needs to be explored more fully especially in the area of sporting activity, primarily because so few participation variables appeared to be connected with this axis in Bennett et al.'s research.

1.8 Exploring these patterns is facilitated by drawing upon Young's phenomenological account of female sporting participation entitled 'Throwing like a Girl'[2] (Young 1990). Bourdieu's failure to sufficiently explore gendered class positions creates a conceptual blind-spot in understanding how distinction is enacted across the social space. Young argues that for the lived body 'the world appears as the system of possibilities... and resistances' (1990: 149) that can be adopted and iterated. For women it is common for these possibilities and resistances to create an environment where women fail 'to make full use of the body's spatial and lateral potentialities' (Young 1990: 145) ${ }^{[3]}$. Accepting Young's insights into the female bodily experience provides a context for appreciating gendered differences within the social space. Thus women may experience a general disposition to minimize the spatiality of their bodily movement but this will be negotiated within the class disposition of instrumentality, for example, if the women is also working class (Young 1990). Consequently, it is expected that there will be gender differences related to the body and that women might be more inwardly oriented than men, as suggested by Bennett et al. (2009). Such theoretical and empirical indicators suggest that this research will observe a variety of complex interactions between gender, sport and class.

1.9 In addition to issues concerning gender, Bennett et al.'s research also draws attention to the role of education in their analysis. They find that higher levels of educational attainment are closely linked with increased levels of sporting participation. The same is also accurate for measures of class. They posit that such a shift is the result of an increased emphasis upon body-maintenance, as discussed earlier. Bourdieu, like Bennett et al., observes that participation in sport increases with class and education; and yet this is not of primary interest to Bourdieu. It is the qualitative differences that interests Bourdieu (1984: 212-3): thus it is imperative to explain why the working classes might be more prone to engage in sports that require a high level of energy, pain or danger. Contrastingly, the middle classes focus on health, training the body and resisting the ageing process. Similarly, for Bourdieu, team sports are, by their nature, inclusive and therefore tend to be rejected by 'elite' groups. Associated with these patterns are the necessities of having the time and opportunity to learn these practices (that are often obtained whilst a youth) which serves to exclude those who do not have such time later in life.

1.10 Finally, sports participation may also be related to friendship. Although those 'with fewer 
qualifications and working-class occupations' tend to have lower levels of cultural engagement (Bennett et al. 2009: 71) they do have rich social and community associations. Bennett et al.'s discussion of the motivations to participate in sport ignores the potential impact of friendship on sports participation, which has been observed in studies of team sports in particular (Hargreaves 1987). Chan \& Goldthorpe (2007) have recently developed a measure of Social Status which is grounded in friendship networks among occupations. They find that those who are high on the status scale (i.e. those whose friendship networks are tightly associated with professional occupations) are more likely to be enthusiastic cultural consumers. They argue that friendship and occupation mediate patterns of cultural consumption. From these research projects it is evident that friendship impacts leisure practices and I expect friendship to be important in this research on sports participation in particular.

1.11 Bourdieu's work, re-read in light of Bennett et al.'s research, presents a series of questions concerning sport and the social strata. Gender, age and social stratification are anticipated to be significant and yet the interconnections between these factors are not evident from previous research. For example, is the increasing emphasis upon body-maintenance tied to notions of middle class respectability or are such notions a reflection of a wider concern across British sporting cultural engagement more generally? Both Bourdieu (1984) and Bennett et al. (2009) observe that the ascetic practice of gym attendance and regulating the body are important practices for the educated middle classes. What are the gender differences in the types of embodied foci that sporting practitioners enact? These questions will be explored in the present research within the British context.

\section{Method}

2.1 This research undertakes multiple correspondence analysis (MCA) of the 68 physical activities listed in the Taking-Part Survey ${ }^{[4]}$ which, using wave one of these data (which was gathered between July 2005October 2006), has a sample size of 28,117. MCA has become well known since Bourdieu used this technique in Distinction (Bourdieu 1984: 128-9, see fig. 5). MCA creates a spatial representation of the relationship between particular variables; if respondents who play football also swim then these will be proximate in this representation of the social space. Likewise if respondents who play football never play golf then these results will be very distant from each other. ${ }^{[5]}$ In order to gather information on sporting activities, the interviewer asked the following question: 'thinking about the last 4 weeks, have you done any sporting or recreation activities?' If the respondent answers 'Yes' then the interviewer asks 'which ones have you done?' (Aust and Vine 2007: 77). Only those activities verbally recalled by the respondent are included in the data.

\section{Constructing the Variables}

2.2 The process of constructing the variables to be included in MCA is vital and defining what sporting variables can be included in the analysis is key (Le Roux and Rouanet 2004; 2010). Like Wittgenstein's (1961) famous illustration of 'games', defining a sport is a difficult task. Though sport is the label used by the survey designers, a better description of the type of practices included on the list is 'physical activities and/or sports'. Activities such as 'horse-riding' or 'fishing', though potentially a 'sport' for some participants, are more likely to be engaged in for the purpose of leisure. Yet, despite this acknowledgement most of the activities listed under this variable would be easily recognisable as a 'sport' even though a specific definition of what that term means might be elusive; which parenthetically seems to be Wittgenstein's principle point in raising this illustration. Consequently every variable present in the data was considered for inclusion and, for brevity, I have continued to use the term sport, even though 'physical activities and/or sports' is more accurate.

2.3 In organising data for MCA it is important that the relative frequencies of the variables selected be above five per cent of the selected sample size. Variables with small frequencies have a larger impact upon the distribution of the categories and can therefore misrepresent the dimensions of the social space (Le Roux and Rouanet 2004). Consequently 'very infrequent categories of active variables... need to be pooled with others whenever feasible' (Le Roux and Rouanet 2010: 39). Three guiding principles directed efforts in organising the variables: first, it was necessary to identify those people who participated in at least one activity over the previous four weeks, second, accurately dividing those variables where frequencies were greater than five per cent and third, providing careful attention to collecting variables into useful categories (i.e. approximately $5 \%$ ). The first principle focused the analysis upon those people who participated in a sport; reducing the available sample size from 28,117 to 14,433 respondents. When the supplementary variables, such as education, were also included in the analysis the sample size was reduced further from 14,433 to 10,349 . Under the second principle, those variables that have frequencies greater than 509 (5\% of 10,349$)$ were included in the analysis as an individual measure of that activity (see Table 1).

2.4 The third guiding principle will be discussed in greater detail because the method of collecting variables together did not include all activities in the data set and represents various levels of specificity. Although this clustering is driven by an empirical concern, the newly created categories are theoretical in that they capture key features of specific physical activities. Table 1 shows the 19 variables eventually included in the analysis. Those variables that have been pooled now have a total frequency which is over $5 \%$. These pooled variables capture logically similar activities, such as yoga and pilates, cycling for health or for mobility, indoor and outdoor bowls. Squash and table tennis are the most dissimilar; however they were grouped together on the basis that they are usually played indoors and with a small number of people and are racket based games (and therefore require a certain degree of skill) ${ }^{[6]}$. In all these categories activities of a similar type have been grouped.

Table 1. List of Constructs and the Survey Variables from which they are formed. 


\begin{tabular}{cl} 
Variable Name & Variables Included \\
Cycling & Cycling Health, Cycling Mobility \\
Bowls & Bowls(indoor), Bowls(outdoor) \\
Snooker/Pool & Snooker/Pool \\
Darts & Darts \\
Badminton & Badminton \\
Swim Outdoor & Swim(outdoor) \\
Swim Indoor & Swim(Indoor) \\
Keepfit & Keepfit (Aerobics, dance exercise, exercise bike) \\
Golf & Golf \\
Yoga/Pilates & Yoga, Pilates \\
Conditioning & Conditioning \\
Bowling & Ten-Pin Bowling \\
Weights & Weight Training, Weightlifting \\
Soccerin & Football(Indoor) \\
Soccerout & Football(Outdoor) \\
Tennis & Tennis \\
Racket & Squash, Table Tennis \\
Fishing & Fishing \\
Running & Running(Cross-country, Jogging or Road-running) \\
\hline
\end{tabular}

\section{Creating Modalities}

2.5 Modalities are the features of MCA that are distributed across the social space and they are created by categorising the responses to specific variables. In this instance each variable is a question (a total of 19) and has two modalities (+, -) creating a total of 38 modalities. Thus anyone who participated in soccer indoors over the last four weeks would have been included in the '+soccerin' modality while all those who have not would have been included in '-soccerin' modality. For those variables where a group of questions were collected together, a respondent would only have to be a participant in one of the categories to have been included in the '+bodybuild' modality for example. This simple binary tries to capture the dual movement of participation and non-participation across those who participate in at least one activity. It is through the (dis)similarity between the patterns of response that the notion of distance is generated. Consequently, if respondents who participate in football ('+soccerin') also participate in darts ('+darts') then these two modalities will be proximate in the social space (Le Roux and Rouanet 2010).

\section{Supplementary Variables}

2.6 A number of supplementary variables were added to the analysis in order to observe the patterns of certain socio-demographic characteristics in relation to the patterns of participation. Such variables are laid over the analysis in order to observe any patterns in how the data is distributed (Le Roux and Rouanet 2004). These supplementary variables do not actively contribute to the structuring of the social space (Greenacre 2006) although they can restrict the sample size available for analysis. As noted earlier, the sample size was further reduced from 14,433 to 10,349 cases because of the inclusion of these variables.

2.7 The supplementary variables used in this analysis include age, social status, educational attainment, gender and class. The age variable was categorised into groups by decade (i.e. 30-39) except for the lowest (17-29) and highest group (70+). ${ }^{[7]}$ Gender is described as male and female. Educational categories include 'Higher Education or equivalent', 'Other Higher Education below degree', 'A Levels or equivalent', 'Trade Apprenticeships', '5> GCSE's', '5< GCSE's' and those with 'Level Unknown'[8]. The class variable followed the nine-category version of the NS-SEC [9]. The measure of Status used here is based upon Chan \& Goldthorpe's scale (2004; Chan 2010) developed using multi-dimensional scaling of friendship data in BHPS. In order to clarify the trends in this scale it has been re-coded into three tiers referring to low, medium and high status.

\section{Results}

3.1 Following a discussion of the number of pertinent dimensions a brief analysis of the non-participants who have not been included in the MCA will be provided. Subsequently, each of the dimensions will be discussed along with those supplementary variables that are significant along that dimension.

3.2 From the Inertia's, or Eigenvalues, (see Table 2), of the MCA four dimensions are considered to be a significant influence upon the cloud of modalities (Le Roux and Rouanet 2010: 52). These four axes are analysed upon the basis that they account for $70 \%$ of the variance among the variables. After this fourth dimension the amount of variance attributable to these different axes (see for example dimensions 5-6 in table 2) are small and the difference between them is minimal. 
Table 2. Principle Dimensions and Inertias from the MCA

$\begin{array}{cccc}\text { Dimension } & \text { Principle Inertia } & \text { Percent } & \text { Cumulative Percent } \\ 1 & .001473 & 40.42 & 40.42 \\ 2 & .000828 & 22.71 & 63.13 \\ 3 & .000173 & 4.74 & 67.87 \\ 4 & .000063 & 1.74 & 69.61 \\ 5 & .000013 & 0.37 & 69.97 \\ 6 & .000009 & 0.09 & 70.06 \\ \text { Total } & .003644 & 100^{*} & \end{array}$

* Not all dimensions are displayed and therefore results do not equal 100 due to rounding.

\section{Non-Participants}

3.3 Because the sample includes only those who participate, some descriptive statistics concerning those non-participants are presented before outlining the results of the MCA proper. $62.2 \%$ of the agricultural labourers and $57.4 \%$ of semi-skilled or unskilled workers are non-participants whilst $66.1 \%$ of professionals and $58.6 \%$ of high-grade non-manual employees are participants. $54.3 \%$ of low-status and $37.9 \%$ of high-status respondents are non-participants. Non-participants are also more likely $(71.1 \%)$ to have few, if any, formal qualifications while $67.1 \%$ of those with the highest educational attainment are likely to be participants. Only $27.7 \%$ of $17-29$ year olds are non-participants compared to $78.9 \%$ of respondents whose age was 70 or above. In addition, $41.6 \%$ of males and $54.4 \%$ of females were nonparticipants suggesting gender differences for non-participation as well. Non-participants are present in all levels of society and there is evidence to indicate that people in lower classes or status groups are more likely to be non-participants. Evidently men aged 17-29 with high levels of education are most likely to participate.

\section{The Four Dimensions}

3.4 In each graph only those modalities which contribute significantly to that dimension are shown. The basic features of each dimension will be described in relation to the various supplementary variables. The first dimension accounts for $42.4 \%$ of the total variance of the cloud of categories (Fig. 1). One of the recurring features of these graphs, and the data more generally, is the clustering of the non-participation modalities ('-darts') in the centre of the graph. This is a reflection of the relatively high frequency with which respondents, even those who do participate in one activity, tend to be non-participants in most other activities. Swimming indoors is the most popular activity and only $28.9 \%$ of the sample participate (14.8\% including the non-participants). Most people do not participate in a wide variety of sporting activities. 


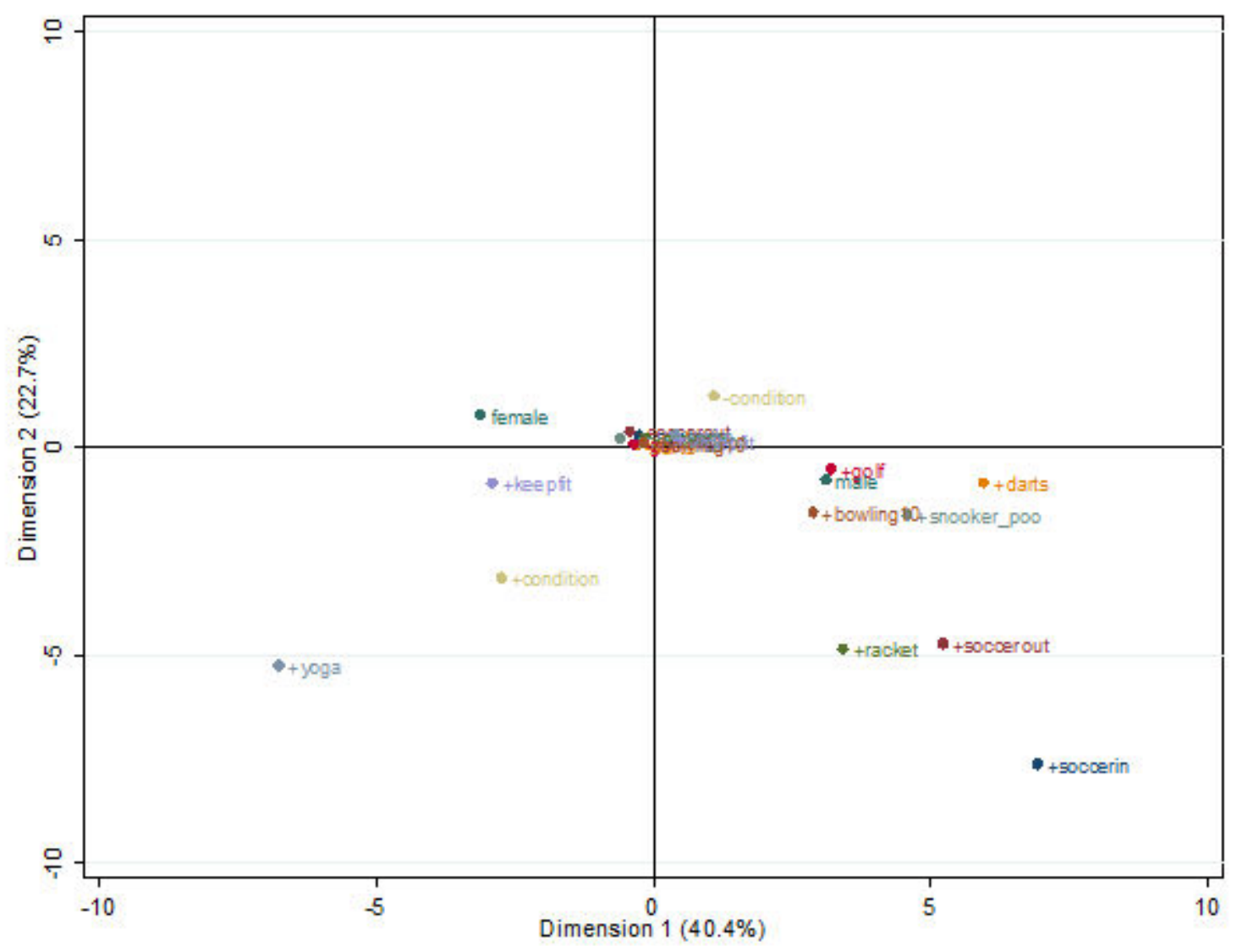

Figure 1. Multiple Correspondence Analysis of Dimension $1 \& 2$ : Coordinates represent modalities with a significant impact upon Dimension 1 with Gender included as a supplemntary variable

3.5 Notably, most of the active modalities on Dimension 1 (see Fig. 1) are on the right side of the space; only the '+yoga', '+condition' and '+keepfit' modalities are on the left-hand side. Figure 1 also includes gender as a supplementary variable along this dimension because it is highly significant.

3.6 Dimension 2, or the vertical axis, accounts for $22.7 \%$ of the total variance of the cloud of categories (Fig. 2). The categories in the top section are '+bowls' and '-condition'. Contrastingly the activities in the bottom section are '+running', '+condition', '+racket', '+soccerin' and '+bodybuild'. The primary supplementary variable on this dimension is Age (Le Roux and Rouanet 2010: 59). Respondents in the 16-29, 30-39 and 40-49 age categories are in the bottom section. Other measures of social stratification are also significant upon this dimension but they are less notable than age. Respondents who have a degree and who work in the service class are more likely to be in the bottom section. 


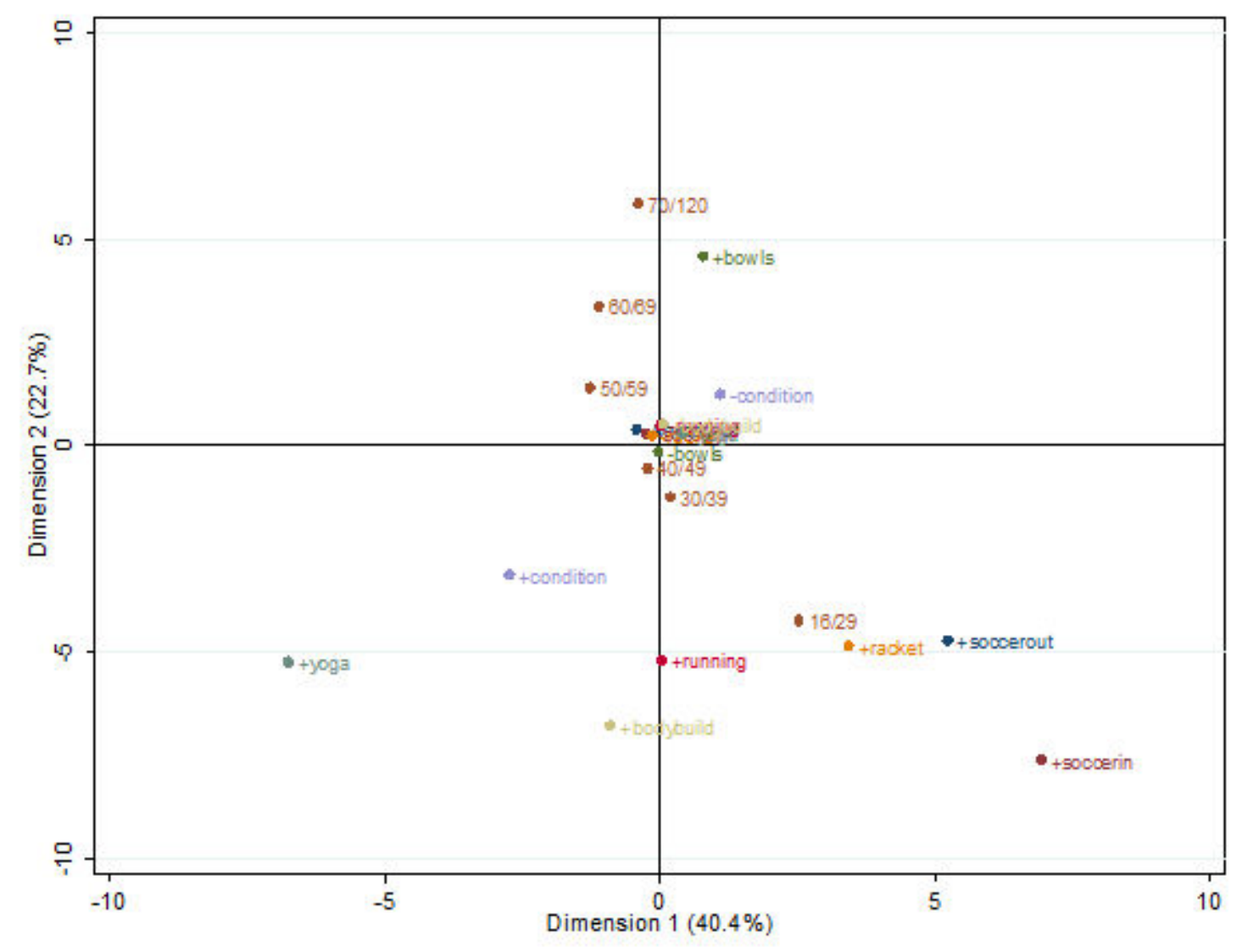

Figure 2. Multiple Correspondence Analysis of Dimension 1 \& 2: Coordinates represent modalities with a significant impact upon Dimension 2 with Age included as supplementary variable.

3.7 The third dimension, the vertical axis, accounts for $4.7 \%$ of the total variance of the cloud of categories (Fig. 3). Those variables in the top section are '+darts', '+snooker/pool'. '+condition' and '+yoga' and the modalities in the bottom section are '+tennis', '+badminton' and '+swimoutdoor'. 


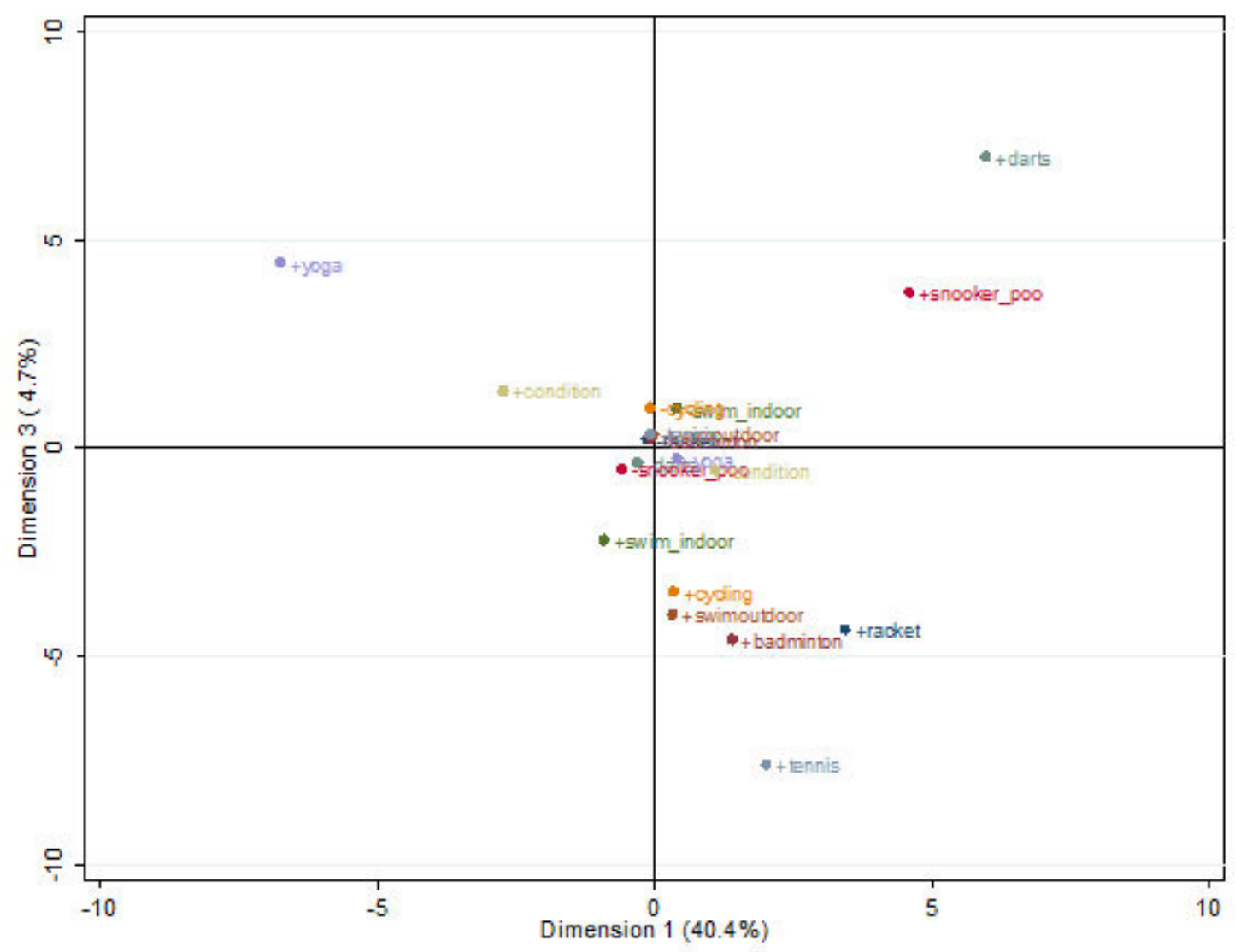

Figure 3. Multiple Correspondence Analysis of Dimension $1 \&$ 3: Coordinates represent modalities with a significant impact upon Dimension 3

3.8 Education, social status and class have a strong linear correlation with dimension 3 (see Fig. 4). For example, professionals, those with a university education and also those with high social status are in the bottom section while those who are with fewer qualifications and those who work in manual occupations are most likely to be in the top section.

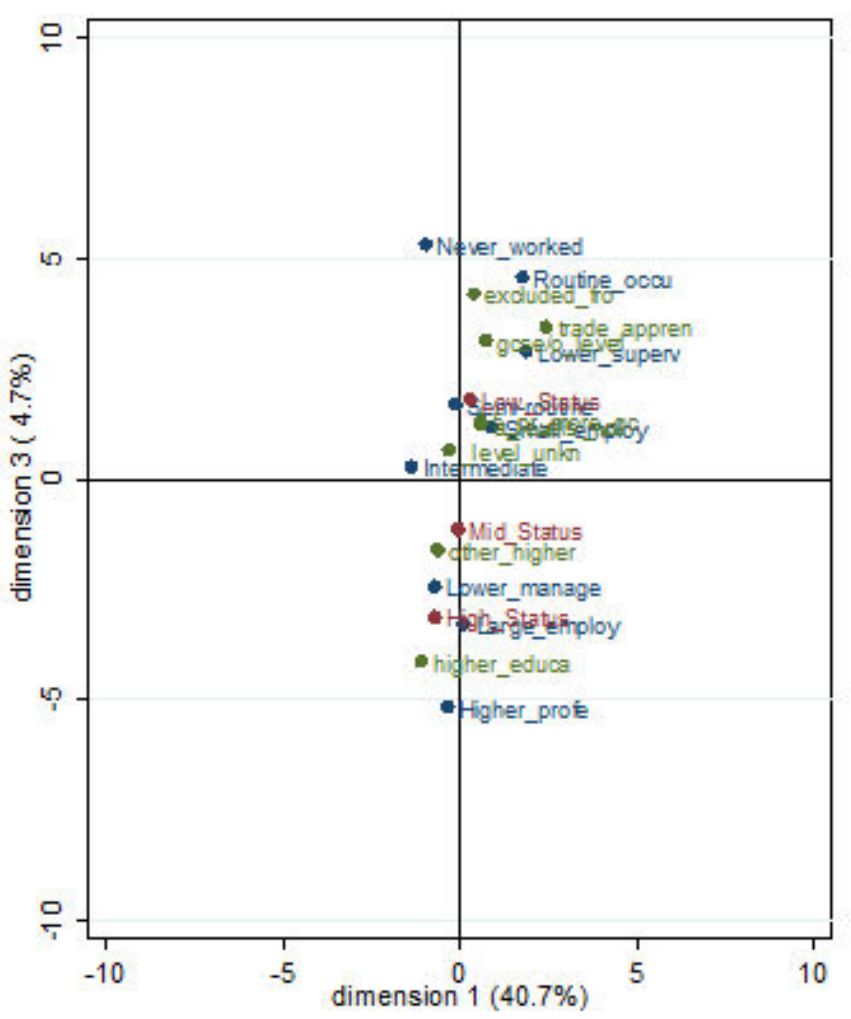

Figure 4. Multiple Correspondence Analysis of Dimension $1 \& 3$ : Coordinates are the modalities of the Education, Social Class and Social Status supplementary variables 
3.9 The data for dimension 4 is difficult to clearly describe and it accounts for $1.7 \%$ of the total variance of the cloud of categories (Fig. 5). Activities such as '+running' and '+bodybuild' are in the top half while '+racket' and '+badminton' are in the bottom. The social stratification variables are strongly correlated with this dimension and it is possible that this is the primary method of deciphering this pattern of distinction. However, there are a number of unexpected results that need further consideration, especially in light of the association between dimension 4 and 3 (see Fig. 6).

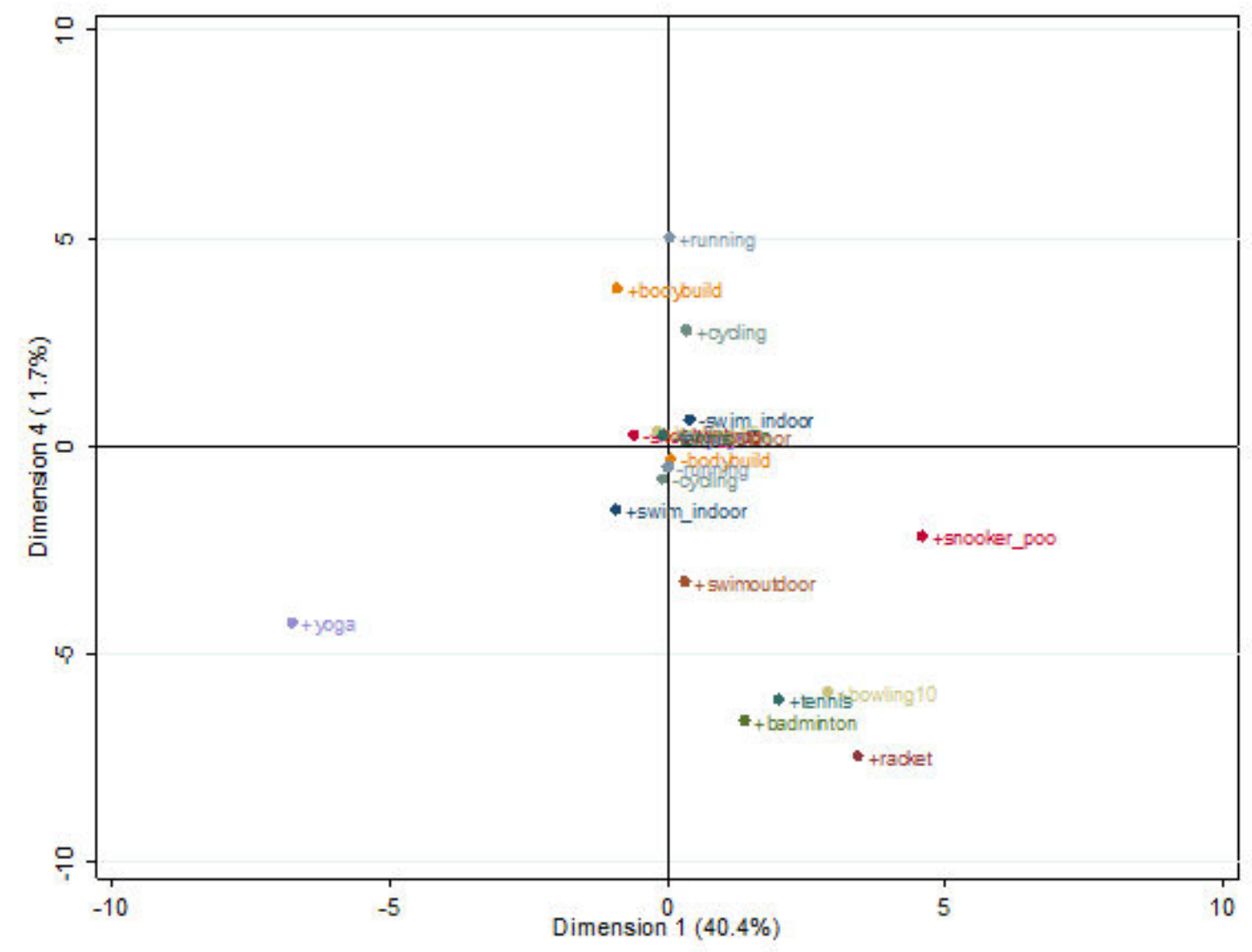

Figure 5. Multiple Correspondence Analysis of Dimension 4 \& 1: Coordinates represent modalities with a significant impact upon Dimension 4

\section{Discussion}

\section{Dimension 1: Embodied and Social Focus}

4.1 Gender, as a supplementary variable, was highly significant on dimension 1. The '+yoga', '+condition' and '+keepfit' modalities are on the left-hand side of the graph and most of the other activities are on the right. Previous research has confirmed that men and women differ in terms of their sporting preferences, although there is some overlap (Bennett et al. 2009). This research finds that this is also accurate for sporting participation. It is important to remember that these positions in the social space represent tendencies and not discrete differences. With that said, it is evident that certain sports (namely yoga, soccer, snooker/pool and darts) serve as markers of gender. Although enacting gender is closely tied to embodied practice of this kind it does not negate the multiple gendered positions available to individuals, as Brown suggests (2006). Another notable feature of the divide between men and women is the number of activities on each side of the line: Women only have three and men, in contrast, have seven. This lack of breadth in female sporting participation (at least among the variables measured here) might be connected with the bodily dis-ease that Young (1990) posits.

4.2 The categories in the male-oriented activities - on the right-side of dimension 1 - also involve participating with others in a communal setting. Contrastingly the female-oriented activities - on the leftside of dimension 1 - focus upon body maintenance. This is not to suggest that those activities on the male side of the graph ignore the body or that those activities on the female side lack sociality. Rather the collection of activities as a whole suggest that an embodied-focus is prevalent among women while a focus on social interaction is prevalent for men. As anticipated, friendship motivates participation; but friendship, in terms of sport, is more important for men. Bennett et al.'s interview data found that women, when discussing their motivation for sports participation, most often cited relaxation or escapism while, somewhat surprisingly, issues concerning the body are not cited at all. Evidence also indicates that female sports participants are more concerned with the body than non-participants (Swami et al. 2009). If women tend to be more anxious about their physique (Mulazimoglu-Balli et al. 2000) then this anxiety may translate into an embodied focus; a focus which also serves as a form of relaxation or escapism from this particular form of anxiety. 
4.3 These gender differences persist across socially stratifying variables. Although the social stratification modalities were not as strongly correlated along this dimension they were still significant (Le Roux and Rouanet 2004). In short, those respondents who have a university degree or who are members of the service class or even those who are high status are more likely to have this embodied focus. This focus is therefore not merely the province of all women but educated or professional women. This theme will be explored more fully later but it is important to recognise here that relations to the body are mediated through class and gender. This reinforces Young's phenomenological approach to female embodied experience and also reflects Bourdieu's intuition concerning the interrelation of class and gender.

4.4 Therefore, the primary conclusion from this dimension is that embodiment and friendship are key features of how sport participation is organised. Further, it is clear that approaches to embodiment and friendship are mediated through gender and class. Bennett et al. (2009) have already noted the importance of bodily maintenance in their discussions of sporting practice yet they seem to neglect friendship and this is problematic. They recognise that for cultural non-participants friendship is important but they fail to appreciate how it might shape patterns of cultural (sporting) practice. Further, these data is consonant with the view that people with few qualifications, or who are unskilled and low-status are more likely to exhibit this socially-focussed orientation in their sporting practice.

4.5 What is unclear from the data available here is how this social focus might be tied to notions of classed subjectivity (Day 2001; Skeggs 2004a; 2005). Skeggs and Day have argued that specific practices (such as body-maintenance) display signs of control, rather than excess (Brown 2005). Therefore, certain socially focused practices could be considered crude or excessive (Skeggs 2004a). Neat distinctions between those practices aimed at body-maintenance and gender are not possible from this data but there are indications that the symbolic value of specific activities is embedded within a variety of contexts. This requires further research.

4.6 This embodied-social dimension invites comparison with the class-based bodily relation of Bourdieu (1984). In his view, the primary difference between classes, in terms of sporting practice, was the disposition exhibited by each class toward the body. Those among the working classes were instrumental while those in the dominant classes displayed an ascetic relation with the body. However, without adequately considering how gender might shape these class-based dispositions, especially in light of the data here, demonstrates that Bourdieu's analysis of gender and class, in relation to sporting practice, is insufficiently nuanced to appreciate the patterns of practice exhibited in the British case.

\section{Dimension 2: Age}

4.7 Along dimension 2 age was highly significant, the youngest age category is at the bottom and the oldest at the top of Figure 2. This suggests that age and gender intersect in organising the social positions of sport practitioners. Moreover, '+bowls' is the only positive activity in the top section of the graph (see Fig. 2). While in the bottom section there are seven activities. Being youthful is strongly correlated with various physical activities which reduces gradually with age. Moreover, it is telling that the only activity that increases with age ('+bowls') is insignificant upon dimension 1 (Scherger 2009), indicating that it is gender neutral. There is a sense in which gender is no longer enacted through physical activity in the same way that it is when a person is 20 years old, for example.

4.8 Bourdieu observed that certain class positions seek to resist the effects of aging through participation in sports. Unsurprisingly various measures of social stratification are also important along this dimension. Educated professionals are more likely to be found in the bottom section, for example. More than just maintaining health these age-resistant sports actually symbolise youth and vitality and they have lower retirement ages. In fact, what may be important on this dimension are those variables which are absent. Badminton, Tennis and Swimming, for instance, are clearly available to a wide range of age groups, because they are not significant along this dimension, but they are also associated with educated professionals. Thus, the primary conclusions from this dimension are the move toward gender neutrality during the aging process and the age-resistant dispositions of certain class positions.

\section{Dimension 3: Internal \& External}

4.9 Education, social status and class are highly significant along dimension 3. Graduates, professionals or high status individuals are most likely to be found in the bottom section of dimension 3 . Those activities in the top section are all enacted inside specific buildings or designated spaces (see Fig. 3). Contrastingly the modalities in the bottom section (i.e. swimoutdoor, tennis and cycling) are often practiced in open spaces. Consequently this dimension appears to be capturing a distinction between an indoor-orientation and an outdoor-orientation toward sporting participation.

4.10 Accepting this definition of the dimension, professionals, those with a degree and those who are high status tend to be externally-oriented. Additionally, those in the low status category tend to have an internal focus for their sporting participation. Moreover, the internally-focused activities are heavily divided across gender: '+yoga' and '+condition' are internal-female while '+darts' and '+snooker/pool' are internalmale while all those activities in the externally-focused section (with the exception of '+racket') are not significant on dimension 1 and therefore are not significantly gender segregated. Implied in these data is a shift in terms of how sport is enacted at different points in the social strata. Low-status, manual occupations or those with fewer qualifications are more likely to engage in activities which are sharply divided by gender whereas gender neutral practices are more common among those who are high-status, professional or have a degree.

4.11 This dimension does not account for as much of the variance as the previous two dimensions but these results indicate an important feature of the social space of sport participation. Although, as noted above, Bourdieu's account of gender and class is problematic, especially in trying to apply it to contemporary British society, there is still a sense in which 'a class is defined... by the place and value it 
gives to the two sexes and their socially constituted dispositions' (Bourdieu 1984: 107). Brown's effort to re-think Bourdieu's theory of gender taps into something that can be valuable in thinking about gender, class and sport. Certainly these dimensions are capturing the potential positions that can be assumed in relation to certain clusters of activity. However these clusters are not, or need not necessarily be, static. Consequently in laying out how gender positions are relational, Brown reinforces something integral to Bourdieu's relational sociology (Savage et al. 2005).

4.12 From this perspective, gender, class and sport (or the specific clusters of sport that are associated with gender and class) become markers of difference. Skeggs (2005) has analysed these forms of difference through notions of value. She writes that one of her research concerns is focused upon 'how bodies, people and groups attain value through different systems of symbolic exchange', and with 'the evaluation of particular bodies and practices' (Skeggs 2005: 46). Where culture is seen as a commodity, or a source of value, Skeggs argues, it becomes possible for a specific practice to be valued by one group whilst simultaneously being devalued when it is attached to another group. Of specific interest here is how this applies to those similarly external activities which might not accrue cultural value for participants who are in different educational or class positions.

4.13 It might be surprising to see Yoga, Pilates or Conditioning in the quadrant dominated by low-status women. However, Skeggs argues that those activities need not necessarily serve the same function for those different groups that practice them. Thus it is clear from these data that none of these groups are discrete. In fact, it is this overlapping, or borrowing, in Skeggs' view, that provides the context for an 'intimate form of exploitation' (2005: 63). Skeggs' argues that there are mechanisms by which middleclass cultural practices seek to re-appropriate, for their own cultural advantage, the practices of other social groups. Deploying this argument here indicates that certain dispositional coalitions (i.e. between body and external) are intimately associated with positions of economic power in the social space. The embodied focus can be blended with those overlapping activities, such as '+yoga' - a variable situated in the internal-body quadrant. In this case vastly different meanings could be attributed to that practice by skilled manual workers or professionals. Beauty, ascetics, escapism and relaxation might be used as a means of articulating these differences, for example. Hence, despite efforts of local authorities to make sports activities of all varieties more accessible, inequalities and distinction are still potentially enacted in these settings. More research is needed to explore whether Skeggs argument applies to these sporting practices. The major conclusions from this dimension are the internal and external focus in activities coupled with the gender-neutrality of the external focus and the recurring intersection between gender, class and sport.

\section{Dimensions 3 \& 4: Social Strata}

4.14 The three measures of social stratification are all significant along dimension 4, with graduates, professionals or high status individuals on the left of the graph. Because the impact of dimension 4 upon the overall cloud of categories is rather small and because the various measures of social stratification are highly significant (but in different ways) on both dimensions 3 and 4 I will consider these dimensions together here in light of the previous discussion of dimension 3. Initially it is quickly apparent that almost all the same variables which are significant on dimension 3 are significant upon dimension 4 (eight out of eleven are significant in both dimensions). 


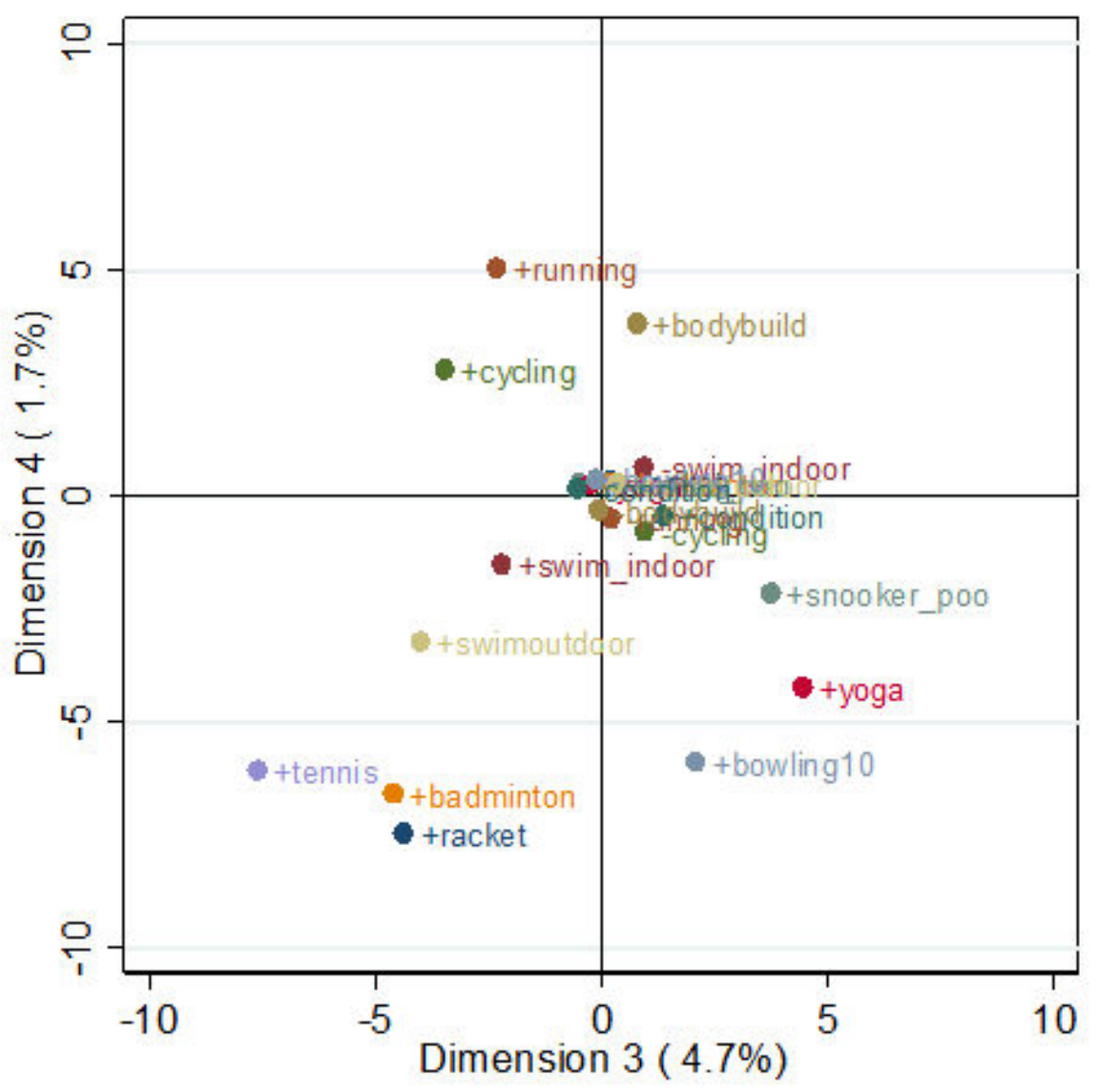

Figure 6. Multiple Correspondence Analysis of Dimension 3 \& 4: Coordinates represent modalities that have a signficant impact upon Dimensions 3 and 4.

4.15 In Figure 5 dimension 3 was the $y$-axis while in Figure 6 dimension 3 is the $x$-axis. Because of this, for example, '+tennis' and '+badminton' are on the left-hand side of Figure 6 and '+snooker/pool' and '+yoga' are on the right, reflecting the vertical axis in Figure 3. 


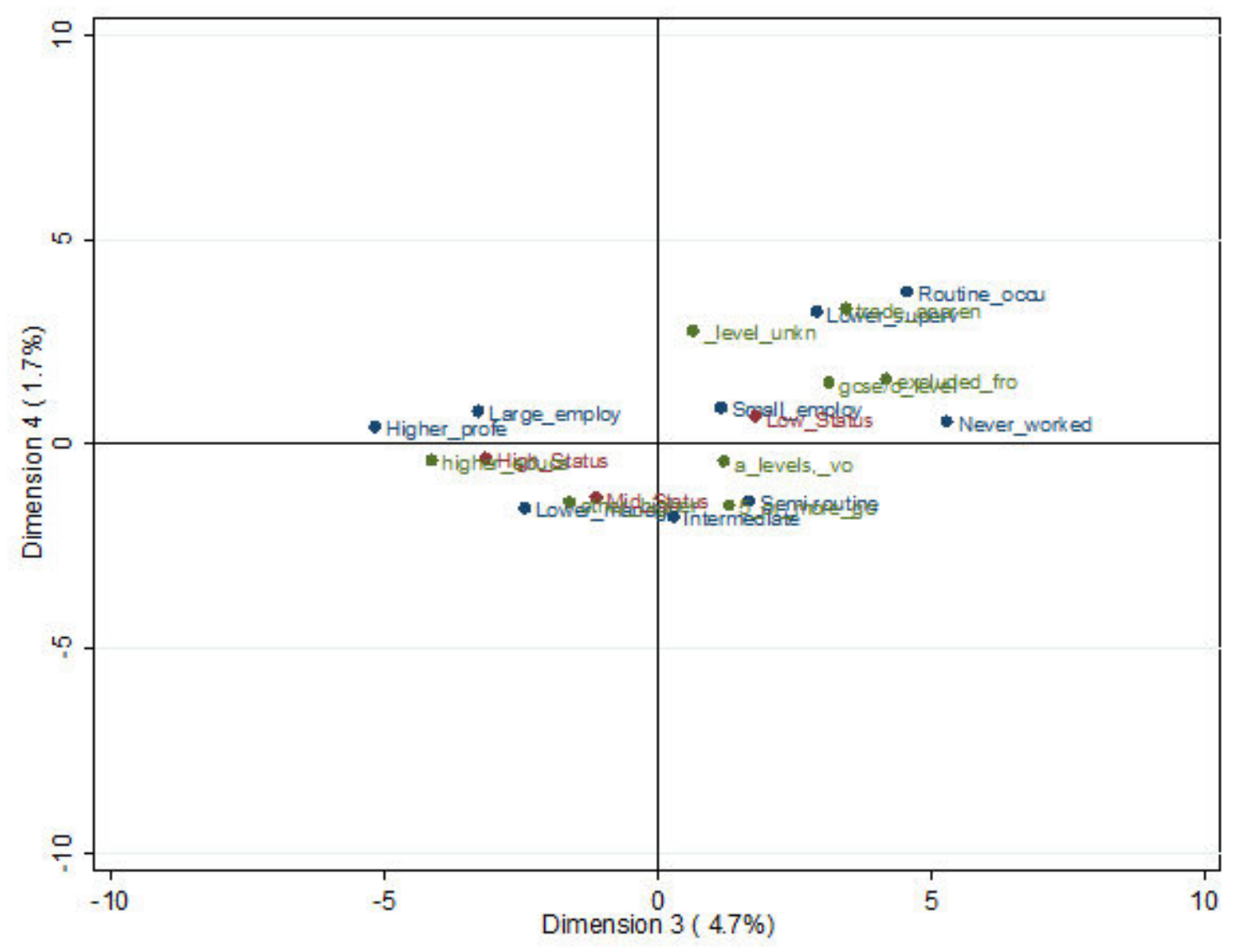

Figure 7. Multiple Correspondence Analysis of Dimension $3 \& 4$ : Coordinates represent supplementary modalities for Education, Social Class and Social Status

4.16 Figure 7 shows the social stratification supplementary variables on dimensions 3 and 4 . Here there are three key clusters. Respondents who are professional, have a degree and are high status are on the left of the graph and therefore significant on dimension 3 but they are not influenced by dimension 4 (because they are close to the horizontal line). In the bottom-right quadrant of Figure 7 , the cluster consists of those who are semi-routine, intermediate occupations and those who have some A Levels or GCSE's. In the upper-right quadrant the cluster consists of those who have little or no qualifications, are low-status and work in manual occupations. Dimension 4 therefore captures a division between those with A Levels or a large number of GCSE's and those without these qualifications; it also represents a difference between those who are intermediate occupations and those who are manual occupations.

4.17 Savage has argued that, occupational distinctions draw the gaze of class awareness horizontally; 'between yourself and others in similar situations' (Savage 2000: 159). People draw comparisons with those close to them in the social strata. These results reflects Savage's argument but they also temper it by suggesting that this dynamic is not overly significant. Like dimension 3 demonstrated, sporting practice serves to distinguish between those who are in similar social class positions.

4.18 That professionals and those with high-status are positioned on the divide along dimension 4 suggests that they have the capacity to move between these different types of activity. This capacity alludes to Skeggs' aforementioned position that, derived from Bourdieu (1984), the same activity is not necessarily pursued with the same intention. However, in terms of sporting participation, it would be spurious to argue that these professionals are a kind of sporting 'omnivore' (Peterson and Kern 1996). Rather they represent small clusters of sporting participation which consistently overlap. Of the sampled respondents, $37.9 \%$ have participated in 2 or 3 activities in the last month and $45.5 \%$ have participated in only 1 . Thus Professionals may be open to participating in a wide variety of sports (see the left-hand section of Figure 6) but they only participate in a few of those possible activities.

4.19 One additional feature of this final dimension might capture what Bourdieu calls 'ease' or a kind of technical familiarity with the activity. Those sports that are linked with a certain bodily-ease often require training, usually in a person's youth, and are specifically related to their schooling (Bourdieu 1978). Thus, tennis or badminton require a high degree of skill which, if absent, can betray a lack of familiarity and reinforce the sense of embodied alienation from participation (Bourdieu 1984). Often these activities involve specifically selected partners who also have these skills. From this view the activities in the bottom section require a greater degree of technical skill. However, in another sense this argument is difficult to maintain because the association between technical skill and exclusivity, which is very explicitly laid out in Bourdieu's work, does not necessarily apply to this data (Bourdieu 1984: 214). For example, 10-pin bowling might not be considered an exclusive sport, especially in the UK where bowling leagues are not popular. Other research needs to consider in greater depth the patterns observed in the data here. From this dimension is the apparent horizontal gaze between specific class positions.

\section{Conclusion}


$\mathbf{5 . 1}$ In the lead up to the 2012 Olympics and with the British governments' efforts to encourage people to 'move more', through the Department of Health's 'Change 4 Life' scheme, sport has been a focal point of the policy agenda (DoH 2009). This paper has provided a more detailed analysis of the social space of sport in Britain than has been previously available by using a wider range of sporting variables and by focusing specifically on sporting participation. It has also articulated a number dimensions that specifically relate to this field of activity. The principal conclusion from this research is that the social space of sporting participation is complex and reciprocal. It is clear that discrete categories of participation are not observable on any of the primary dimensions discussed here; the results suggest the likelihood of participation among certain groups of individuals based upon specific demographic variables. In this instance a more relational approach to cultural engagement is appropriate, as articulated by Savage et al. (2005) in their discussion of Bourdieu. Consequently, any government scheme intending to increase sporting participation will need to be sensitive to the intersection of these various dimensions.

5.2 Although this research moves on the debate regarding the role of sport in the UK, this research has also raised a number of questions that need to be explored in further research. Firstly, these same issues need to be examined using other methodologies. Although MCA represents the relationality of particular activities in the social space, it does so at the expense of obscuring other features of the data. It is more difficult, for example, to pin down the precise correlation between age and football using this approach. Additionally, because of the clustering of certain activities together (and the exclusion of some all together) this research has not examined all possible forms of sport participation present in contemporary British society. This lacuna suggests that specific attention be given to those activities excluded here.

5.3 Secondly a number of potential research questions have been outlined in the paper thus far. Because of low frequencies in some of the variables, there was a necessity to collate some variables into imperfect categories. This has reduced the complexity of the available data. Due to inadequate data on ethnicity, despite a booster sample, issues surrounding race/ethnicity have not been considered in any depth at all. This is an important dimension which requires future consideration (Bennett et al. 2009). Finally it is evident that additional research is needed to trace how sporting practices are tied to notions of the self, value and social stratification.

5.4 Although gender and age are the primary factors along which sporting participation is structured these are strongly mediated by social stratification. This is a recurring theme throughout the data. In terms of sports participation, gender and age cannot, and perhaps should not, be separated from class when considering the processes by which people practice sport. Second, as anticipated, the strong gender divide in sporting preference is also observed for sporting participation. Data from this research indicates that being sensitive to the gendered distinction between an embodied or social focus illuminates the ways in which sport serves to position individuals in relation to other social group. Ageresistant practices are adopted by educated professionals and sport participation becomes increasingly gender neutral as people mature. Educated professionals have an external approach to sport which is also more gender neutral than the internal approach adopted among manual workers with fewer qualifications. In addition there is also evidence, though the impact is not as significant as on other dimensions, that there is a horizontal gaze between the middle and the working classes. Sport participation is evidently still a locus of social conflict.

\section{Notes}

${ }^{1}$ The social space is one representation of the relationship between a set of variables. Dimensions are the factors which influence the position of those variables in the representation of the social space (Le Roux and Rouanet 2000). Thus if those people who play tennis are close to those who play golf in the graphical representation of the social space they are close only in that dimension

${ }^{2}$ Young (1990) is aware that her account is not universal even within the relatively small location from which her observations have been derived. It is thus an attempt to outline general trends that can be observed across many women. Moreover her account is not supposed to be a comprehensive discussion of all forms of bodily movement but is instead a specific account of bodily comportment and of intentional action. Both Merleau-Ponty (1967) and De Beauvoir (1972) are significant precursors to her work.

\footnotetext{
${ }^{3}$ Moreover, 'women tend not to put their whole bodies into engagement in a physical task' (1990: 145), they tend to be passive in sports (allowing the ball to come to them) and there is a lack of trust toward the body that women experience. This lack of trust might be reflected in an increased focus upon training the body it an attempt to reclaim this lack.

${ }^{4}$ The survey data consists of face-to-face interviews with people aged 16 or over in private households in England. For each wave population weighting has been used in accordance with population figures obtained from the Office for National Statistics and a booster sample of adults from non-white backgrounds was also introduced to an otherwise random sample that was stratified by 'region, population density and proportion of residents classified as managerial/professional/full-time students' (Aust and Vine 2007: 104).
}

\section{${ }^{5}$ When thinking about MCA a few important details are important to remember. First, MCA is used for categorical variables. Second, 'clouds' are part of the specific nomenclature of MCA. The cloud refers to the cluster of reference points of each modality in graph. Third, each cloud point is like a geographic} map. The same distance scale is used in all directions (Le Roux and Rouanet 2000).

${ }^{6}$ Further, from an empirical point of view, when each activity was included in the analysis as a separate variable the coordinates in the social space were very similar, despite being exaggerated. On this basis, therefore, I argue that they capture people who have similar sporting dispositions and can therefore be 
reliable included in the analysis.

${ }^{7}$ These age categories have been selected following Bennett et al. (2009), who also used similar agegroups in their multiple correspondence analysis of cultural practice in the UK.

${ }^{8}$ In the England, Northern Ireland and Wales, GCSE is an acronym for 'General Certificate of Secondary Education'. These are usually received for specific subjects, such as Mathematics, when pupils complete their compulsory schooling aged 16 . Those students who receive 5 GCSE's graded $A^{*}-C$ (the four highest grades) are considered to have received an adequate education. Following compulsory schooling a variety of options are available; students can receive formal training in a skilled trade through an apprenticeship or they can pursue academic schooling by taking 'A Levels' (Advanced Level General Certificate of Education). A Levels are usually completed when students are aged 18. Higher education refers to any university level degree and 'other Higher education' pertains to training which is equivalent to a university degree but is (often) vocational, or work-based. The level unknown category, though included in the analysis, was not interpreted as part of the results primarily because it is not possible to infer from this category any of the qualities of its members.

${ }^{9}$ NS-SEC is an acronym for 'National Statistics Socioeconomic Classification'. It was developed in conjunction with Goldthorpe's class schema (Chan and Goldthorpe 2004).

\section{Acknowledgements}

I am grateful to the Economic and Social Research Council for funding this research. For helpful comments and advice, I thank the three anonymous reviewers and the conference participants at the Leisure Studies Association's annual conference in Southampton.

\section{References}

ADKINS, L. and SKEGGS, B. (2004). Feminism after Bourdieu. Oxford, Blackwell.

ANTHIAS, F. (2005). Social Stratification and Social Inequality: Models of Intersectionality and Identity. Rethinking Class: Culture, Identities and Lifestyle. M. Savage, J. Scott, R. Compton and F. Devine. New York, Palgrave MacMillan: 24-45.

AUST, R. and VINE, L. (2007) "Taking Part: The National Survey of Culture, Leisure and Sport."

BENNETT, T., SAVAGE, M., SILVA, E., WARDE, A., GAYO-CAL, M. and WRIGHT, D. (2009). Culture, class, distinction. London, Routledge.

BOURDIEU, P. (1978). "Sport and Social-Class." Social Science Information Sur Les Sciences Sociales 17(6): 819-840. [doi:://dx.doi.org/10.1177/053901847801700603]

BOURDIEU, P. (1984). Distinction : a social critique of the judgement of taste. London, Routledge \& Kegan Paul.

BOURDIEU, P. (2001). Masculine domination. Cambridge, Polity.

BOURDIEU, P. (2008 [1999]). "A Conservative Revolution in Publishing." Translation Studies 1: 123-153. [doi:://dx.doi.org/10.1080/14781700802113465]

BROWN, D. (2006). "Pierre Bourdieu's "Masculine Domination" thesis and the gendered body in sport and physical culture." Sociology of Sport Journal 23(2): 162-188.

BROWN, J. A. (2005). "Class and Feminine Excess: The Strange Case of Anna Nicole Smith." Feminist Review 81: 74-94. [doi:://dx.doi.org/10.1057/palgrave.fr.9400240]

BUNTING, C., CHAN, T. W., GOLDTHORPE, J. H., KEANEY, E. and OSKALA, A. (2008). From Indifference to Enthusiasm: Patterns of Arts Attendance in England. London, Arts Council England.

CERON-ANAYA, H. (2010). "An Approach to the History of Golf: Business, Symbolic Capital, and Technologies of the Self." Journal of Sport \& Social Issues 34(3): 339-358.

[doi:://dx.doi.org/10.1177/0193723510377317]

CHA, V. D. (2009). "A Theory of Sport and Politics." International Journal of the History of Sport 26(11): 1581-1610. [doi:://dx.doi.org/10.1080/09523360903132972]

CHAN, T. W. (2010). Social status and cultural consumption. Cambridge, Cambridge University Press. [doi:://dx.doi.org/10.1017/CBO9780511712036]

CHAN, T. W. and GOLDTHORPE, J. H. (2004). "Is there a status order in contemporary British society? Evidence from the occupational structure of friendship." European Sociological Review 20(5): 383-401. [doi:://dx.doi.org/10.1093/esr/jch033]

CHAN, T. W. and GOLDTHORPE, J. H. (2007). "Social status and newspaper readership." American Journal of Sociology 112(4): 1095-1134. [doi:://dx.doi.org/10.1086/508792]

CONNELL, R. (1995). Masculinities. Cambridge, Polity. 
CONNELL, R. W. and MESSERSCHMIDT, J. W. (2005). "Hegemonic Masculinity: Rethinking the Concept." Gender \& Society 19(6): 829-859. [doi:://dx.doi.org/10.1177/0891243205278639]

COULANGEON, P. (2004). "Social classes, cultural practices and lifestyles: Is the distinction model (really) obsolete?" Sociologie et societies 36(1): 59-85.

COULANGEON, P. and LEMEL, Y. (2009). The Homology Thesis: Distinction Revisited. Quantifying Theory: Pierre Bourdieu. K. Robson and C. Sanders. London, Springer: 47-60.

CROOK, C. J. (1997). "The dimensionality of stratification-related cultural practices in Australia." Australian and New Zealand Journal of Sociology 33(2): 226-238.

[doi:://dx.doi.org/10.1177/144078339703300207]

DAY, G. (2001). Class. London, Routledge.

DE BEAUVOIR, S. D. and PARSHLEY, H. M. (1972). The second sex. Harmondsworth, Penguin. DCMS (2010). Putting Sport into People's Lives: Sport Legacy in Action. London, Crown Copyright. $\mathrm{DOH}$ (2009). Be Active, Be Healthy: A plan for getting the Nation moving. London, HM Government.

DOLLMAN, J. and LEWIS, N. R. (2010). "The impact of socioeconomic position on sport participation among South Australian youth." J Sci Med Sport 13(3): 318-322. [doi:://dx.doi.org/10.1016/j.jsams.2009.04.007]

GREENACRE, M. (2006). From Simple to Multiple Correspondence Analysis. Multiple Correspondence Analysis and Related Methods. M. Greenacre and J. Blasius. London, Chapman \& Hall: 41-76.

HARGREAVES, J. (1987). Sport, Power and Culture: A Social and Historical Analysis of Popular Sports in Britain. Oxford, Blackwell Publishers.

KRAIS, B. (2006). "Gender, Sociological Theory and Bourideu's Sociology of Practice." Theory, Culture and Society 23(6): 119-134. [doi:://dx.doi.org/10.1177/0263276406069778]

LAHIRE, B. (2010). The plural actor. Cambridge, Polity.

LAMONT, M. L. (1992). Money, morals, and manners : the culture of the French and American uppermiddle class. Chicago ; London, University of Chicago Press.

LAW, J. (2007). Making a Mess with Method. The Sage Handbook of Social Science Methodology. W. Outhwaite and S. P. Turner. Beverly Hills and London, Sage: 595-606.

LE ROUX, B. and ROUANET, H. (2004). Geometric data analysis : from correspondence analysis to structured data analysis. Dordrecht ; London, Kluwer Academic.

LE ROUX, B. and ROUANET, H. (2010). Multiple correspondence analysis. London, SAGE.

LOPEZ-SINTAS, J. and GARCIA-ALVAREZ, E. (2006). "Patterns of audio-visual consumption: The reflection of objective divisions in class structure." European Sociological Review 22(4): 397-411. [doi:://dx.doi.org/10.1093/esr/jcl004]

MAYS, N. (2008). Origins and Development of the National Health Service. Sociology as Applied to Medicine. G. Scambler. London, Saunders: 221-251.

MCNAY, L. (1999). "Gender, Habitus and the Field: Pierre Bourdieu and the limits of Reflexivity." Theory, Culture \& Society 16(1): 95-117.

MERLEAU-PONTY, M. (1967). Phenomenology of perception. London, Routledge and Kegan Paul, .

MULAZIMOGLU-BALLI, O., KOCA, C. and ASCI, F. H. (2010). "An Examination of Social Physique Anxiety with Regard to Sex and Level of Sport Involvement." Journal of Human Kinetics 26: 115-122. [doi:://dx.doi.org/10.2478/v10078-010-0055-9]

PETERSON, R. A. and KERN, R. M. (1996). "Changing highbrow taste: From snob to omnivore." American Sociological Review 61(5): 900-907. [doi:://dx.doi.org/10.2307/2096460]

ROWE, N., ADAMS, R. and BEASLEY, N. (2004). Driving Up Participation In Sport - the social context, the trends, the prospects and the challenges. Driving up participation: the challenge for sport. . London, Sport England.

SAVAGE, M. (2000). Class analysis and social transformation. Buckingham, Open University.

SAVAGE, M., WARDE, A. and DEVINE, F. (2005). "Capitals, assets, and resources: some critical issues." British Journal of Sociology 56(1): 31-47. [doi:://dx.doi.org/10.1111/j.1468-4446.2005.00045.x]

SCHERGER, S. (2009). "Cultural practices, age and life-course." Cultural Trends 18(1): 23-45. [doi:://dx.doi.org/10.1080/09548960802651203]

SKEGGS, B. (2004a). Class, self, culture. London, Routledge.

SKEGGS, B. (2004b). "Context and Background: Pierre Bourdieu's analysis of class, gender and 
sexuality." Feminism After Bourdieu: 19-33.

SKEGGS, B. (2005). The re-branding of class: Propertising culture. Rethinking Class: Culture, Identities and Lifestyles. F. Devine, J. Scott, M. Savage and R. Crompton. Basingstoke, Palgrave Macmillan: 46-68.

STALSBERG, R. and PEDERSEN, A. V. (2010). "Effects of socioeconomic status on the physical activity in adolescents: a systematic review of the evidence." Scandinavian Journal of Medicine \& Science in Sports 20(3): 368-383. [doi:://dx.doi.org/10.1111/j.1600-0838.2009.01047.x]

SWAMI, V., STEADMAN, L. and TOVEE, M. J. (2009). "A comparison of body size ideals, body dissatisfaction, and media influence between female track athletes, martial artists, and non-athletes." Psychology of Sport and Exercise 10(6): 609-614. [doi:://dx.doi.org/10.1016/j.psychsport.2009.03.003]

WARDE , A. (2006). "Cultural Capital and the Place of Sport." Cultural Trends 15(2/3): 107-122.

WASHINGTON, R. E. and KAREN, D. (2001). "Sport and society." Annual Review of Sociology 27: 187212. [doi:://dx.doi.org/10.1146/annurev.soc.27.1.187]

WITTGENSTEIN, L. (1961). Tractatus logico-philosophicus. New York,, Humanities Press.

YOUNG, I. M. (1990). Throwing like a girl and other essays in feminist philosophy and social theory by Iris Marion Young. Bloomington, Ind., Indiana University Press. 